\title{
Distributed Control of Networked Agent Systems: Theory and Applications
}

\author{
Guanghui Wen, ${ }^{1}$ Haibo Du, ${ }^{2}$ Chaojie Li, ${ }^{3}$ Qiang Song, ${ }^{4}$ and Wenwu Yu${ }^{1}$ \\ ${ }^{1}$ Research Center for Complex Systems and Network Sciences, School of Mathematics, Southeast University, Nanjing 210096, China \\ ${ }^{2}$ School of Electrical Engineering and Automation, Hefei University of Technology, Hefei 230009, China \\ ${ }^{3}$ School of Engineering, RMIT University, Melbourne, VIC 3001, Australia \\ ${ }^{4}$ College of Electronic Engineering, Henan University of Technology, Zhengzhou 450001, China
}

Correspondence should be addressed to Guanghui Wen; wenguanghui@gmail.com

Received 31 May 2017; Accepted 1 June 2017; Published 12 July 2017

Copyright (C) 2017 Guanghui Wen et al. This is an open access article distributed under the Creative Commons Attribution License, which permits unrestricted use, distribution, and reproduction in any medium, provided the original work is properly cited.

A great number of practical complex systems can be modeled as networked agent systems. Typical examples include the distributed satellite systems, a group of robots, wireless sensor networks, and power grids. One critical topic within this context is to understand how globally cooperative behaviors of such systems can be emerged as a result of distributed local interactions which has recently received much attention from various scientific fields.

The present special issue mainly focuses on the new distributed control approaches in networked agent systems as well as their potential engineering applications. It tries to not only explore the underlying mechanisms corresponding to various collective behaviors but also manipulate and even control these fascinating collective behaviors. Call for papers has been carefully prepared by the guest editors and posted on the journal's web page, which has received a lot of attention from researchers in various scientific fields. This special issue has received 30 submissions on networked agent systems. All manuscripts submitted to this special issue went through a thorough peer-refereeing process. Based on the reviewers' reports and the guest editors' comments, 10 original research articles are finally accepted. The contents of accepted papers are summarized below.

(a) Distributed Control and Optimization of Networked Agent Systems. The paper "On Couple-Group Consensus of Multiagent Networks with Communication and Input Time Delays" by L. Ji and X. Zhao investigates couple-group consensus/synchronization of multiagent systems. Some new sufficient criteria for reaching couple-group consensus have been presented. The work of "Distributed Optimization of Multiagent Systems in Directed Networks with Time-Varying Delay" by J. Yan and H. Yu designs a continuous-time distributed optimization algorithm to solve the distributed optimization problem of first-order multiagent systems. In "Synchronization of Coupled Harmonic Oscillators Using Quantized Sampled Position Data" by X. Wang and P. He, the synchronization problem of networked harmonic oscillators with quantized current sampled position data is studied. In "Plug and Play Robust Distributed Control with Ellipsoidal Parametric Uncertainty System" by W. Hong and Y. Wang, distributed control of a continuous linear time invariant system with ellipsoidal parametric uncertainty structured into subsystems is studied.

(b) Analysis and Synthesis of Complex Dynamical Systems. In "Iterative Learning Control with Forgetting Factor for Urban Road Network" by T. Lan et al., to improve the traffic condition, a new iterative learning control (ILC) algorithm with forgetting factor for urban road network is proposed by using the repeat characteristics of traffic flow. The paper "Parameters Design for Logarithmic Quantizer Based on Zoom Strategy" by J. Yan investigates the problem of designing suitable parameters for logarithmic quantizer such that the closed-loop linear time-invariant discrete system is asymptotically convergent. In "A Novel Measurement Matrix 
Optimization Approach for Hyperspectral Unmixing" by $\mathrm{S}$. $\mathrm{Xu}$ and $\mathrm{X}$. He a novel approach for the optimization of measurement matrix in compressive sensing theory for hyperspectral unmixing is proposed. In "An Out Space Accelerating Algorithm for Generalized Affine Multiplicative Programs Problem" by L. Cai et al., an out space branchand-bound algorithm is designed for solving generalized affine multiplicative programs problem. The paper "An Effective Algorithm for Globally Solving Sum of Linear Ratios Problems" by $\mathrm{H}$. Jiao et al. proposes an effective algorithm for globally solving the sum of linear ratios problems. In "DCA-Based Real-Time Residual Useful Life Prediction for Critical Faulty Component" by F. Zhou et al., to predict the residual useful life effectively, wavelet filtering based method is developed for early detection of slowly varying fault of dynamical systems.

\section{Acknowledgments}

We would like to thank the authors for their contributions and acknowledge all the reviewers for their time and effort in assessing the manuscripts. Special thanks to the Editorial Office of this journal for their kind support.

Guanghui Wen Haibo Du Chaojie Li Qiang Song Wenwu Yu 


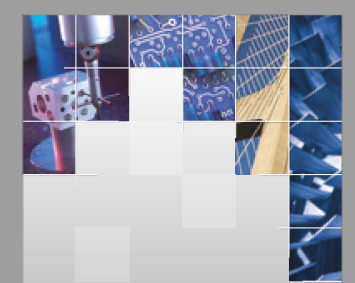

\section{Enfincering}
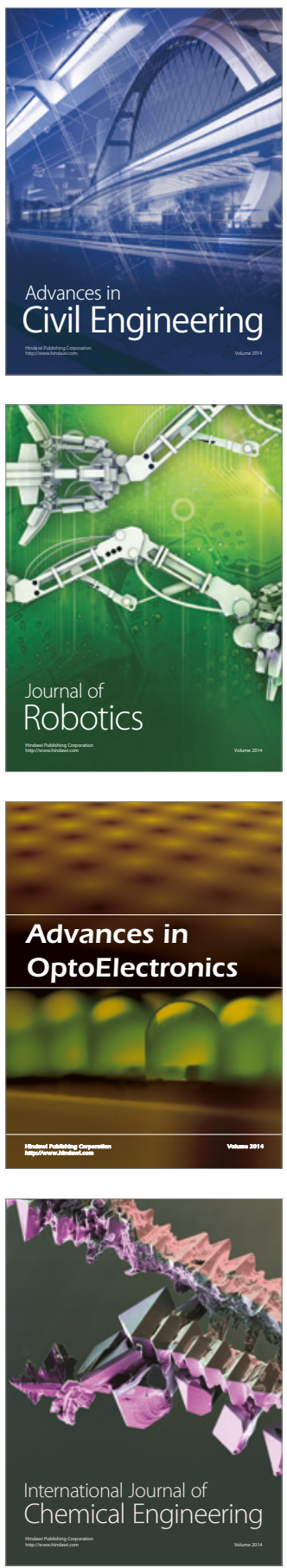

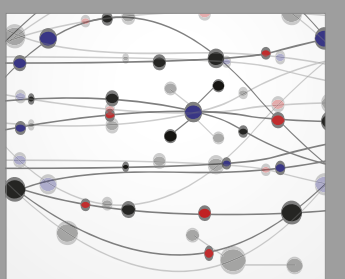

The Scientific World Journal

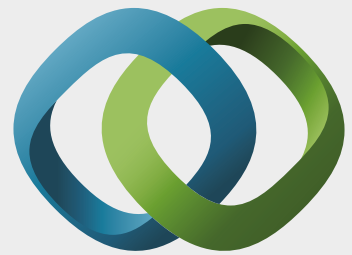

\section{Hindawi}

Submit your manuscripts at

https://www.hindawi.com
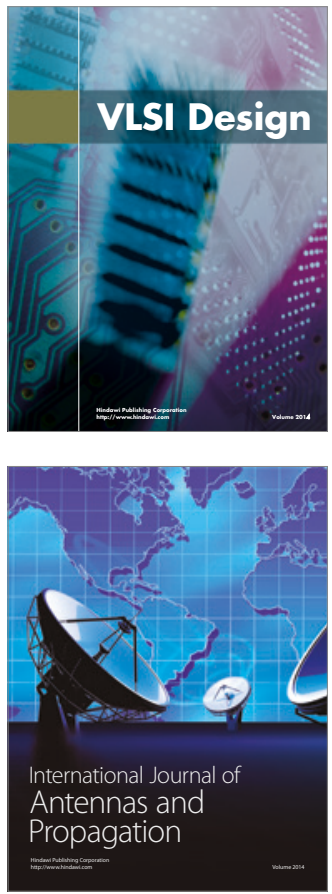

\section{Rotating}

Machinery
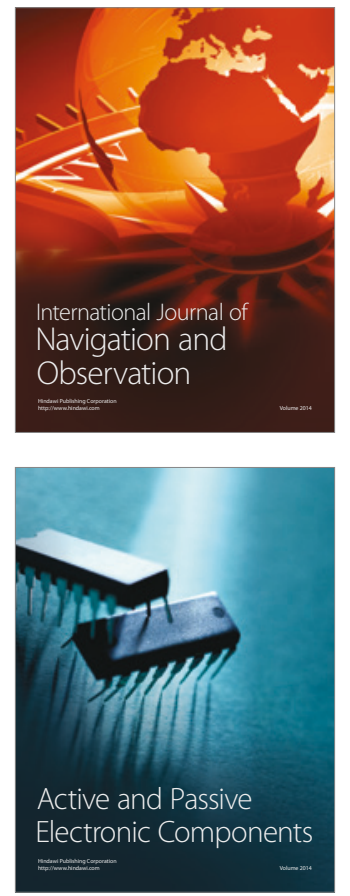
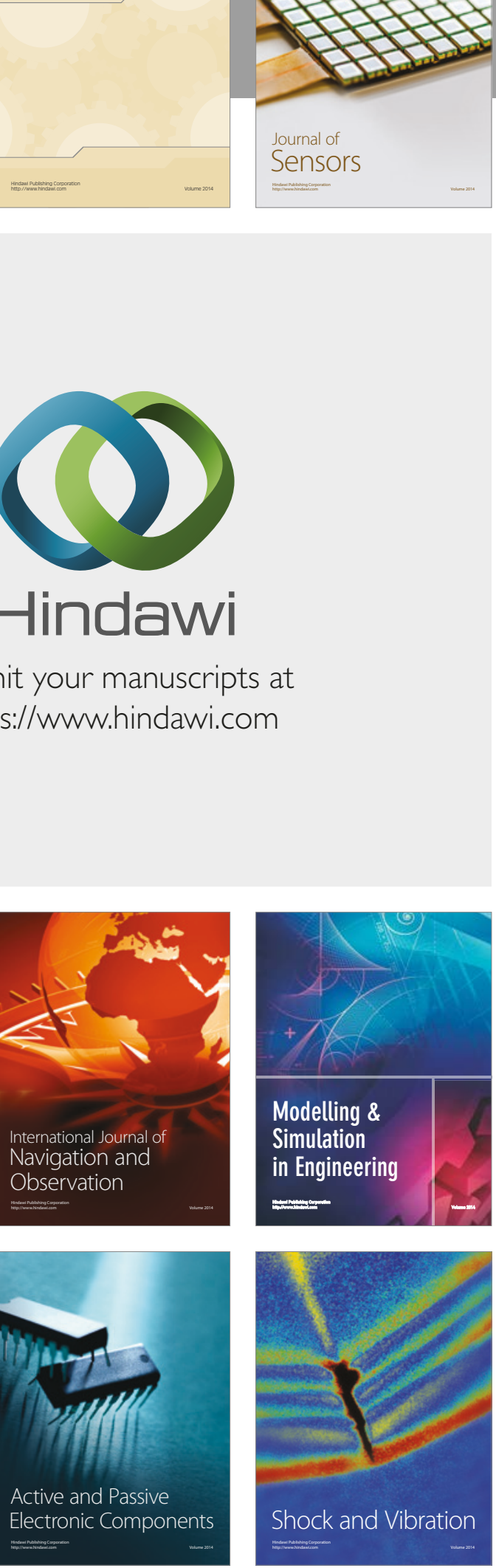
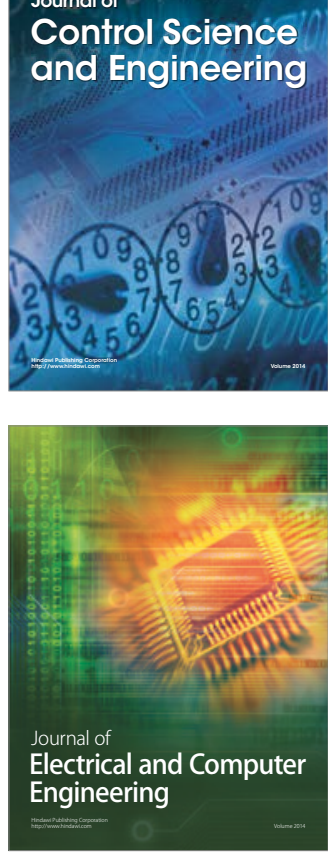

Distributed

Journal of

Control Science

and Engineering
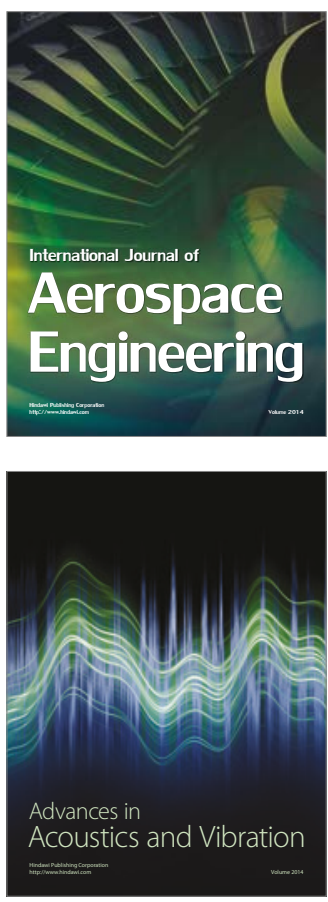

Sensor Networks 